\title{
AN AREA THEOREM FOR A ONE-DIMENSIONAL SEMIDIRECT EXTENSION OF HOMOGENEOUS GROUPS
}

\author{
EWA DAMEK
}

(Communicated by David G. Ebin)

\begin{abstract}
Let $N$ be a homogeneous group [3] and let $\left\{\delta_{a}: a \in A=R^{+}\right\}$be the group of dilations of $\dot{N}$. We prove an area theorem for harmonic functions w.r.t. a class of second-order left-invariant hypoelliptic differential operators $L$ on the semidirect product $S=N A$ with $a x a^{-1}=\delta_{a}(x), a \in A, x \in N$.
\end{abstract}

Introduction. Area theorems have been proved for the harmonic functions on the half-space [8] and for the harmonic functions w.r.t. the Laplace-Beltrami operator on symmetric spaces: rank-one case in [5], the product of two rank-one spaces in [6].

In [5] A. Korányi and R. Putz have proved that, for a harmonic function $f$ on a symmetric space $G / K, G=\bar{N} A K$ and a measurable set $M \subset \bar{N}$ the following are equivalent:

(i) $f$ is a.e. nontangentially bounded on $M$;

(ii) the area integral is a.e. finite on $M$;

(iii) $f$ is a.e. nontangentially covergent on $M$.

In the present paper we prove the implication (i) $\rightarrow$ (ii) in a more general situation.

We start with an arbitrary homogeneous group $N$ with dilations $\left\{\delta_{a}: a \in A=\right.$ $\left.R^{+}\right\}$and we form the semidirect product $S=N A, x a x^{\prime} a^{\prime}=x \delta_{a}\left(x^{\prime}\right) a a^{\prime}, x, x^{\prime} \in N$, $a, a^{\prime} \in A$. We equip $S$ with a left-invariant Riemannian metric in such a way that if $N$ comes from the Iwasawa decomposition, then $S$ becomes a rank-one symmetric space. As in [2] we consider not only the Laplace-Beltramni operator but a large class of second-order left-invariant hypoelliptic operators. In the case of a symmetric space $G / K$ this covers second-order hypoelliptic operators which commute with the $\bar{N} A$-action and anihilate constants. Since all the left-invariant Riemannian metrics on $S$ give proportional volume elements, the final result depends only on the group structure not on the Riemannian metric.

The method used in the present paper is a modification of the one of A. Korányi and R. Putz [5]. We have replaced the mean value theorem for symmetric spaces by Harnack inequality [1] and the Green theorem by the divergence theorem.

Whether the remaining implications hold in the case considered here is an open question. The proof by A. Korányi and R. Putz in [5] is based on very specific properties of symmetric spaces and the Laplace-Beltrami operator and we do not see any method of replacing it by another argument.

Received by the editors November 20, 1987.

1980 Mathematics Subject Classification (1985 Revision). Primary 22E30; Secondary 43A85.

Key words and phrases. Area theorem, left-invariant operators, homogeneous groups. 
Area theorem. A simply connected nilpotent Lie group $N$ is called homogeneous [3] if there is a basis $E_{1}^{\prime}, \ldots, E_{n}^{\prime}$ of the Lie algebra $\underline{n}$ of $N$ and numbers $1=d_{1} \leq \cdots \leq d_{n}$ such that for $a>0$ the map $E_{j}^{\prime} \rightarrow a^{d_{j}} E_{j}^{\prime}$ extends to an automorphism $\delta_{a}$ of $\underline{n}$. Identifying $X$ and $\exp X$ we see that $\delta_{a}$ is an automorphism of $N$. It is called a dilation.

On $N$ there is a function

$$
N \ni x \rightarrow|x| \in R^{+}
$$

(homogeneous norm), which is $C^{\infty}$ outside $x=e, \delta_{a}(x)=a|x|,|x|=0$ iff $x=e$, $|x y| \leq \gamma(|x|+|y|)$ for some $\gamma \geq 1$. If meas denotes the Lebesgue measure on $N$ and $B(r)=\{x \in N:|x|<r\}$, we have meas $(B(r))=c_{1} r^{Q}$ for a universal constant $c_{1}$ and $Q=d_{1}+\cdots+d_{n}$.

In this paper we study the solvable group $S=N A$ which is the semidirect product of $N$ and the group of dilations $A=R^{+}$with $a x a^{-1}=\delta_{a}(x), a \in A, x \in N$. Let $E_{1}, \ldots, E_{n}$ be left-invariant vector fields on $S$ corresponding to $E_{1}^{\prime}, \ldots, E_{n}^{\prime}$ and $E_{0}$ a vector field defined by

$$
E_{0} f(s)=\left.\frac{d}{d t} f\left(s e^{t}\right)\right|_{t=0}, \quad e^{t} \in A, s \in S .
$$

Then $E_{j}$ applied to the function $S \ni s \rightarrow a(s)^{Q} \subset R^{+}$is equal to

$$
\begin{array}{cl}
Q a(s)^{Q} & \text { if } j=0 \text { and to } \\
0 & \text { otherwise. }
\end{array}
$$

We define a Riemannian metric $\langle$,$\rangle on S$ assuming that $E_{0}, \ldots, E_{n}$ are orthonormal. The volume element $\eta$ is then proportional to the left-invariant measure on $S$, that is to $a^{-Q-1} d x d a$.

If $N=R, n=1, d_{1}=1$ we obtain the hyperbolic plane-the simplest example of a symmetric space. In fact the hyperbolic plane may be identified with the group

$$
S=R \times R^{+}=\left\{(x, a): x \in R, a \in R^{+}\right\},
$$

where

$$
(x, a)\left(x_{1}, a_{1}\right)=\left(x+a x_{1}, a a_{1}\right)
$$

and the left-invariant metric is $a^{-2} \delta_{i j}$. In this case $E_{0}=\partial / \partial a, E_{1}=a \partial / \partial x$ and $\eta=a^{-2} d x d a$.

Applying the formula

$$
\left\langle\nabla_{Y_{1}} Y_{2}, Y_{3}\right\rangle=\frac{1}{2}\left(\left\langle\left[Y_{1}, Y_{2}\right], Y_{3}\right\rangle-\left\langle\left[Y_{2}, Y_{3}\right], Y_{1}\right\rangle+\left\langle\left[Y_{3}, Y_{1}\right], Y_{2}\right\rangle\right)
$$

for the Riemannian connection $\nabla$ (cf. eg. [7]) we have

$$
\nabla_{E_{i}} E_{i}=d_{i} E_{0} \text {. }
$$

Since $(\operatorname{div} Y)_{s}=\operatorname{tr}\left(w \rightarrow \nabla_{w} Y\right), w \in T_{s} S$,

$$
\operatorname{div} Y=\sum_{i=0}^{n} E_{i} \varphi_{i}-Q \varphi_{0},
$$

where $Y=\sum_{i=0}^{n} \varphi_{i} E_{i}, \varphi_{i} \in C^{\infty}(S)$. As in [5] we define the regions

$$
\Gamma_{\alpha}^{\tau}(x)=\left\{y a:\left|y^{-1} x\right|<\alpha a, a<\tau\right\}
$$


and we write

$$
W_{\alpha}^{\tau}(M)=\bigcup_{x \in M} \Gamma_{\alpha}^{\tau}(x)
$$

for a measurable set $M \subset N$. We say that a function $f$ is nontangentially bounded at $x$ if there are $\alpha, \tau>0$ such that $f$ is bounded in $\Gamma_{\alpha}^{\tau}(x)$.

Let $L$ be a second-order left-invariant operator $L$ on $S$ of the form

$$
L=\left(E_{0}+X_{0}\right)^{2}+X_{1}^{2}+\cdots+X_{m}^{2}+X
$$

where $X_{0}, X_{1}, \ldots, X_{m} \in \underline{n}$ and $E_{0}+X_{0}, X_{1}, \ldots, X_{m}, X$ generate the Lie algebra of $S$. We say that a function $f$ is harmonic iff $L f=0$. We write

$$
D(f)=\left(\left(E_{0}+X_{0}\right) f\right)^{2}+\left(X_{1} f\right)^{2}+\cdots+\left(X_{m} f\right)^{2} .
$$

The aim of this paper is the following

THEOREM. If $f \in C^{\infty}(S)$ is harmonic and nontangentially bounded at almost every point of a measurable set $M \subset N$, then for every $\alpha, \tau>0$

$$
\int_{\Gamma_{\alpha}^{\tau}(x)} D(f) d \eta<\infty
$$

for a.e. $x \in M$.

We may assume that $M$ is bounded. The proof of the Theorem is based on four lemmas. The first is a simple modification of Lemma 5 of [4], the second has been proved in [5].

LEMMA 1. Let $\alpha, \beta, \sigma, \varepsilon$ be positive numbers and $M$ a measurable set. There are $\tau>0$ and a measurable set $D \subset M$, meas $(M \backslash D)<\varepsilon$ such that

$$
\Gamma_{\alpha}^{\tau}(y) \subset \bigcup_{x \in M} \Gamma_{\beta}^{\sigma}(x)
$$

for $y \in D$.

LEMMA 2. Let $M$ be a measurable set. If $\int_{W_{\alpha}^{r}(M)} D(f) a^{Q} d \eta<\infty$ then $\int_{\Gamma_{\alpha}^{\tau}(x)} D(f) d \eta<\infty$ for a.e. $x \in M$.

LEMMA 3. Let $f$ be a bounded harmonic function on $\Gamma_{\beta}^{\sigma}(x)$. Then for every $\alpha<\beta, \tau<\sigma, D(f)$ and $E_{i} f, i=0, \ldots, n$ are bounded in $\Gamma_{\alpha}^{\tau}(x)$.

PROOF. Since $L$ is left-invariant, it is sufficient to consider only the case $x=e$. Let $\Gamma_{\beta}^{\sigma}(e)=\Gamma_{\beta}^{\sigma},|g|_{\infty}=\sup _{x \in \Gamma_{\beta}^{\sigma}}|g(x)|$ and $K=\left\{x a:|x|<\alpha a, \frac{1}{2} \tau<a<\tau\right\}$. Then $b K=\left\{x a:|x|<\alpha a, \frac{1}{2} b \tau<a<\tau b\right\}$ and $\Gamma_{\alpha}^{\tau}=\bigcup_{b<1} b K$. The Harnack inequality [1] implies that there is a constant $c_{2}$ such that for every harmonic function $g$ on $\Gamma_{\beta}^{\sigma}, \sup _{K} D(g)$ and $\sup _{K}\left|E_{i} g\right|, i=0, \ldots, n$, are bounded by $c_{2}|g|_{\infty}$. To estimate $D(f),\left|E_{i} f\right|$ on $b K, b<1$, we take the harmonic function $g(s)=f(b s)$ defined on $b^{-1} \Gamma_{\beta}^{\sigma} \supset \Gamma_{\beta}^{\sigma}$. Then

$$
\sup _{b K} D(f)=\sup _{K} D(g), \quad \sup _{b K}\left|E_{i} f\right|=\sup _{K}\left|E_{i} g\right|, \quad i=0, \ldots, n .
$$

Hence $\sup _{b K} D(f), \sup _{b K}\left|E_{i} f\right|, i=0, \ldots, n$ are bounded by $c_{2}|f|_{\infty}$. 
We write $L$ in the form

$$
L=\sum_{i, j=0}^{n} \alpha^{i j} E_{i} E_{j}+\sum_{i=0}^{n} \alpha^{i} E_{i}, \alpha^{i j}=\alpha^{j i}
$$

and define

$$
L^{*}=\sum_{i, j=0}^{n} \alpha^{i j} E_{i} E_{j}+\sum_{i=0}^{n}\left(-\alpha^{i}-2 Q \alpha^{0 i}\right) E_{i}+Q\left(Q \alpha^{00}+\alpha^{0}\right) .
$$

Then

$$
\int_{S}(L f) g d \eta=\int_{S} f\left(L^{*} g\right) d \eta, \quad f, g \in C_{c}^{\infty}(S)
$$

because

$$
\begin{aligned}
& \int_{S} g E_{i} f d \eta=-\int_{S} f E_{i} g d \eta \quad \text { for } i>0 \text { and } \\
& \int_{S} g E_{0} f d \eta=-\int_{S} f E_{0} g d \eta+Q \int_{S} f g d \eta .
\end{aligned}
$$

Taking into account (2) we can easily check that

$$
f L g-g L^{*} f=\operatorname{div}\left(\sum_{i=0}^{n}\left(\sum_{j=0}^{n} \alpha^{i j}\left(f E_{j} g-g E_{j} f\right)+\left(Q \alpha^{0 i}+\alpha^{i}\right) f g\right) E_{i}\right) \text {. }
$$

The next step is to replace the regions $W_{\alpha}^{\tau}(M)$ by regions $W_{l}$, on which we can apply Stoke's Theorem [9, p. 100]. We take the same regions $W_{l}$ as A. Korányi and R. B. Putz in [5]. Let $\left\{x_{j}\right\}_{j=1}^{\infty}$ be a countable dense sequence in $M$ and

$$
\begin{aligned}
& W_{l}=\bigcup_{1 \leq j \leq l}\left(\Gamma_{\alpha}^{\tau}\left(x_{j}\right) \cap\{x a: a>1 / l\}\right) \\
& B_{l, 0}=\partial W_{l} \cap\{x a: a=\tau\} \\
& B_{l, 1}=\partial W_{l} \cap\{x a: 1 / l \leq a<\tau\}
\end{aligned}
$$

Then $W_{l} \subset W_{l+1}, \bigcup_{l=1}^{\infty} W_{l}=W_{\alpha}^{\tau}(M)$ and we have the following lemma (Lemma 5 of $[5])$.

LEMMA 4. Let $d \rho$ be the surface element on $W_{l}, d / d n$ the outward pointing unit normal to $B_{l, 1}$ and $d n$ the left-invariant volume element on $N$. Then there exist constants $c_{3}, c_{4}>0$ independent of $l$ such that

$$
a^{Q} \leq-c_{3} d a^{Q} / d n, \quad-\left(d a^{Q} / d n\right) d \rho=c_{4} \pi^{*}(d n),
$$

where $\pi: B_{l, 1} \rightarrow N$ by $\pi(x a)=x$.

THE PROOF OF THE THEOREM. Let $\varepsilon>0$ and $M_{m}=\left\{x \in M:|f|,\left|E_{i} f\right|, i=\right.$ $0, \ldots, n, D(f)<m$ on $\left.\Gamma_{1 / m}^{1}(x)\right\}$. Since $M_{m} \subset M_{m+1}$ and by Lemma 3 meas $(M)=$ meas $\left(\bigcup_{m=1}^{\infty} M_{m}\right)$, we can choose $M_{m}$ such that meas $\left(M \backslash M_{m}\right)<\varepsilon / 2$. Let $\alpha>0$, $\tau>0$. By Lemma 1 there is a measurable set $D$ such that meas $\left(M_{m} \backslash D\right)<\varepsilon / 2$ and

$$
|f|, D(f),\left|E_{i} f\right|, \leq m \text { on } \bigcup_{x \in D} \Gamma_{\alpha}^{\tau}(x), \quad i=0, \ldots, n
$$


Now in view of Lemma 2 it is sufficient to show that $\int_{W^{\tau}(D)} D(f) a^{Q} d \eta<\infty$. Since by (1) $L^{*} a^{Q}=0$, we have $2 a^{Q} D(f)=a^{Q} L f^{2}-\left(L^{*} a^{Q}\right) f^{2}$ for a harmonic function $f$. On the other hand (3) combined with (1) gives

$$
a^{Q} L f^{2}-\left(L^{*} a^{Q}\right) f^{2}=\operatorname{div}\left(\sum_{i=0}^{n}\left(\sum_{j=0}^{n} \alpha^{i j} E_{j} f^{2}+\alpha^{i} f^{2}\right) a^{Q} E_{i}\right) .
$$

Applying the divergence theorem

$$
\int_{W_{\iota}}(\operatorname{div} Y) d \eta=\int_{\partial W_{\iota}}\left\langle Y, \frac{d}{d n}\right\rangle d \rho
$$

we obtain

$$
\int_{W_{l}} A^{Q} D(f) d \eta=\frac{1}{2} \int_{\partial W_{l}}\left\langle\sum_{i=0}^{n}\left(\sum_{j=0}^{n} \alpha^{i j} E_{j} f^{2}+\alpha^{i} f^{2}\right) a^{Q} E_{i}, \frac{d}{d n}\right\rangle d \rho .
$$

Hence by (4)

$$
\int_{W_{l}} a^{Q} D(f) d \eta<\frac{1}{2} c_{5} \int_{\partial W_{l}} a^{Q} d \rho
$$

for a constant $c_{5}$ and by Lemma 4 the right side of (5) is uniformly bounded.

\section{REFERENCES}

1. J. M. Bony, Principe du maximum, inégalité de Harnack et unicité du problème de Cauchy pour les opérateurs elliptiques dégénérés, Ann. Inst. Fourier Grenoble 19 (1969), 277-304.

2. E. Damek, Left-invariant degenerate elliptic operators on semidirect extensions of homogeneous groups, Studia Math. 89 (1988), 169-196.

3. G. B. Folland and E. M. Stein, Hardy spaces on homogeneous groups, Princeton Univ. Press, Princeton, N.J., 1982.

4. A. Korányi, Harmonic functions on Hermitian hyperbolic space, Trans. Amer. Math. Soc. 135 (1969), 507-516.

5. A. Korányi and R. B. Putz, Local Fatou theorem and area theorem for symmetric spaces of rank one, Trans. Amer. Math. Soc. 224 (1976), 157-168.

6. _ An area theorem for products of symmetric spaces of rank one, Bull. Soc. Math. France (2) 105 (1981), 3-16.

7. J. Milnor, Curvatures of left-invariant metrics on Lie groups, Adv. in Math. 21 (1976), 293-329.

8. E. M. Stein, On the theory of harmonic functions of several variables. II, Behavior near the boundary, Acta Math. 106 (1961), 137-174.

9. H. Whitney, Geometric integration theory, Princeton Univ. Press, Princeton, N.J., 1957.

Institute of Mathematics, Plac Grunwaldzki 2/4, 50-384 Wroclaw, Poland 